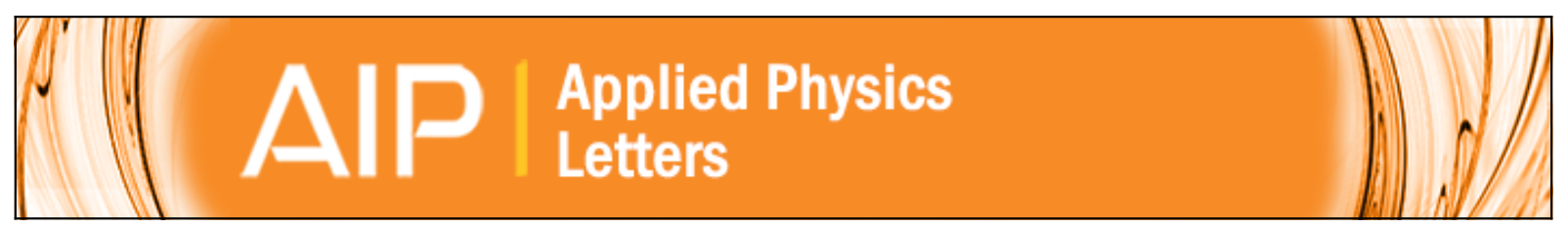

\title{
Ferrite-filled cavities for compact planar resonators
}

P. S. Keatley, C. J. Durrant, S. J. Berry, E. Sirotkin, A. P. Hibbins, and R. J. Hicken

Citation: Applied Physics Letters 104, 022405 (2014); doi: 10.1063/1.4861844

View online: http://dx.doi.org/10.1063/1.4861844

View Table of Contents: http://scitation.aip.org/content/aip/journal/apl/104/2?ver=pdfcov

Published by the AIP Publishing

\section{Articles you may be interested in}

Tuning limitations of the voltage-controlled planar microwave ferrite resonator

J. Appl. Phys. 111, 07 A506 (2012); 10.1063/1.3672397

Electric field tunable ferrite-ferroelectric hybrid wave microwave resonators: Experiment and theory

J. Appl. Phys. 100, 093905 (2006); 10.1063/1.2372575

Electric field tuning characteristics of a ferrite-piezoelectric microwave resonator

Appl. Phys. Lett. 88, 143503 (2006); 10.1063/1.2191950

A grating-assisted resonant-cavity-enhanced optical displacement detection method for micromachined sensors Appl. Phys. Lett. 85, 3032 (2004); 10.1063/1.1804605

Effective microwave ferrite convolver using a dielectric resonator

Appl. Phys. Lett. 81, 1645 (2002); 10.1063/1.1504182

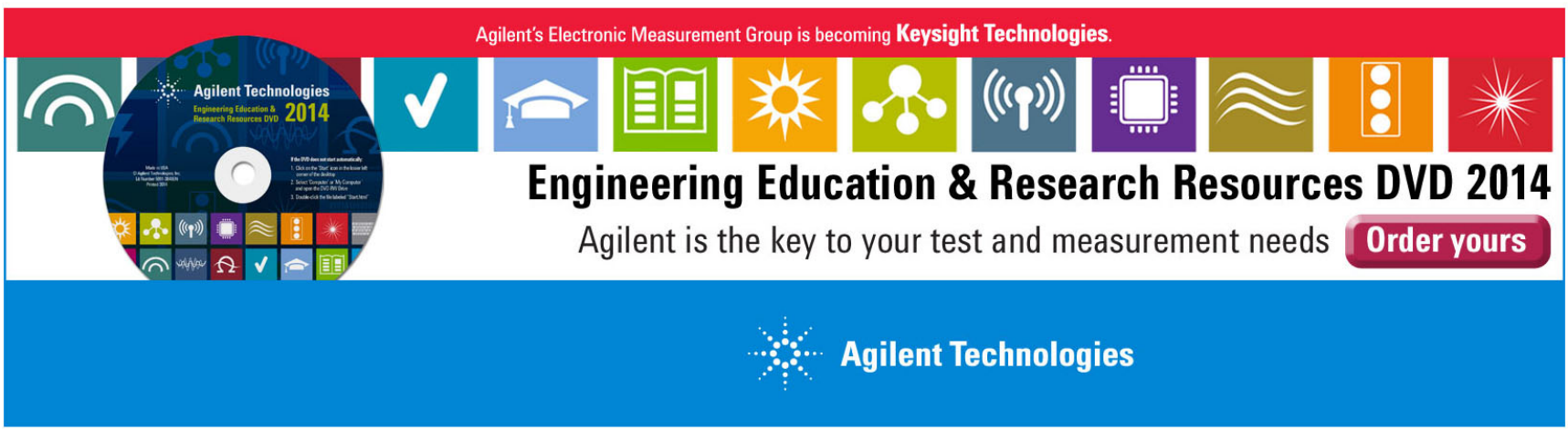




\title{
Ferrite-filled cavities for compact planar resonators
}

\author{
P. S. Keatley, ${ }^{1}$ C. J. Durrant, ${ }^{1}$ S. J. Berry, ${ }^{1}$ E. Sirotkin, ${ }^{1,2}$ A. P. Hibbins,${ }^{1}$ and R. J. Hicken ${ }^{1}$ \\ ${ }^{1}$ School of Physics and Astronomy, University of Exeter, Stocker Road, Exeter EX4 4QL, United Kingdom \\ ${ }^{2}$ School of Engineering, The University of Edinburgh, Faraday Building, The King's Buildings, Mayfield Road, \\ Edinburgh EH9 3JL, United Kingdom
}

(Received 23 October 2013; accepted 26 December 2013; published online 13 January 2014)

\begin{abstract}
Sub-wavelength metallic planar cavities, closed at one end, have been constructed by wrapping aluminium foil around teflon or ferrite slabs. Finite cavity width perturbs the fundamental cavity mode frequency of ferrite-filled cavities due to different permeability inside and outside of the cavity, in contrast to teflon-filled cavities, while the cavity length required to achieve a specific resonance frequency is significantly reduced for a ferrite-filled cavity. Ferrite-filled cavities may be excited by an in-plane alternating magnetic field and may be advantageous for high-frequency (HF) and ultra HF tagging and radio frequency identification of metallic objects within security, manufacturing, and shipping environments. (C) 2014 AIP Publishing LLC.
\end{abstract}

[http://dx.doi.org/10.1063/1.4861844]

The use and exploitation of wireless communication, tagging, and location technologies has advanced rapidly in recent years. However the design of a desirable and portable device places strict miniaturisation requirements on the size of the antenna and favors the use of a sub-wavelength resonator. Sub-wavelength resonators are also the "meta-atoms," of which artificial metamaterials and metasurfaces are comprised. It has been known for many years that a planar array of such meta-atoms can provide a high impedance ground plane that suppresses surface waves and enhances the efficiency and beam pattern of antennas in close (sub-wavelength) proximity. ${ }^{1}$ Improved understanding of planar-coil antennas has been central to the development of cheap and compact radio frequency identification devices (RFID), particularly in the high-frequency (HF) regime $(3-30 \mathrm{MHz})$. However the conventional design, comprised conductive foils or etched copper plating on a plastic substrate, is ineffective when mounted on a conducting material, e.g., a metallic container or a thin dielectric shell containing a polarisable liquid. This is because excitation of the planar coil antenna requires a time varying magnetic field with a component normal to the plane of the coil. Due to Lenz's Law, this perpendicular magnetic field component produces eddy currents within the neighbouring conducting material that generate an equal and opposite magnetic field, inhibiting the excitation efficiency of the tag. The present study is inspired by this problem and provides a compact resonator that can couple efficiently to free-space radiation, without being affected by the conductivity of the material on which it is placed.

It has long been known that two parallel conducting planes separated by an insulator can support a transverse electromagnetic (TEM) waveguide mode, which has no lowfrequency cut-off. ${ }^{2}$ In other words, unlike conventional rectangular cross-sectional waveguide geometries, the TEM mode propagates between the planes no matter how closely they are spaced. The planes may be truncated to form a 1 dimensional cavity of finite length, with air-filled cavities being realized by milling narrow slits in a conducting plate. It was demonstrated that microwave radiation can couple to the TEM mode and that a family of standing wave modes can be excited along the length of such a cavity. ${ }^{3}$ If the plate is a good conductor and the slit is sufficiently narrow, then near complete transmission of microwaves through an array of such slits is observed experimentally at these standing wave resonances. ${ }^{4,5}$ A cavity with two open ends may be described as a half-wavelength $(\lambda / 2)$ resonator, and its transmission response is analogous to that of the Fabry-Pérot resonant optical cavity. This is due to the reflecting boundary condition associated with strong diffraction at each open end of the cavity. ${ }^{6}$ This yields antinodes in the electric field at the open ends of the cavity for a standing wave mode supported by a cavity with length equal to an integer number of half wavelengths. By closing one end of the cavity with a conducting wall, an electric field node is introduced at the wall, and the cavity is instead able to support a $\lambda / 4$ resonant mode.

For an infinitely wide cavity ( $w_{c}=w_{f} \rightarrow \infty$, Figure 1(a)) containing an insulating material, the frequency of the standing wave TEM mode depends upon the length of the cavity and the complex refractive index $n=\left(\varepsilon_{r} \mu_{r}\right)^{1 / 2}$ of the filling material (where $\varepsilon_{r}$ and $\mu_{r}$ are the complex, relative permittivity and permeability). The resonant frequency is given by

$$
\frac{1}{f}=\frac{4 L}{c} \sqrt{\frac{\operatorname{Re}\left(\varepsilon_{r}\right)}{2}}\left\{\left[\left(\operatorname{Re}\left(\mu_{r}\right)\right)^{2}+\left(\operatorname{Im}\left(\mu_{r}\right)\right)^{2}\right]^{\frac{1}{2}}+\operatorname{Re}\left(\mu_{r}\right)\right\}^{\frac{1}{2}}
$$

where $\varepsilon_{r}$ is assumed to be real. While previous studies were largely limited to air-filled cavities or utilised non-magnetic materials with low permittivity, we will show that there is great potential for size reduction by filling the cavity with insulating ferrite with a large and frequency dependent permeability $\mu_{r}$. We construct finite width slit cavities with closed ends using aluminium foil, which is a near-perfect conductor in the microwave- and radio-frequency regimes. This allows the time-varying magnetic field $H(t)$ associated with the surface current $i(t)$ to circulate around the foil and permeate both the surrounding medium (air), where the 


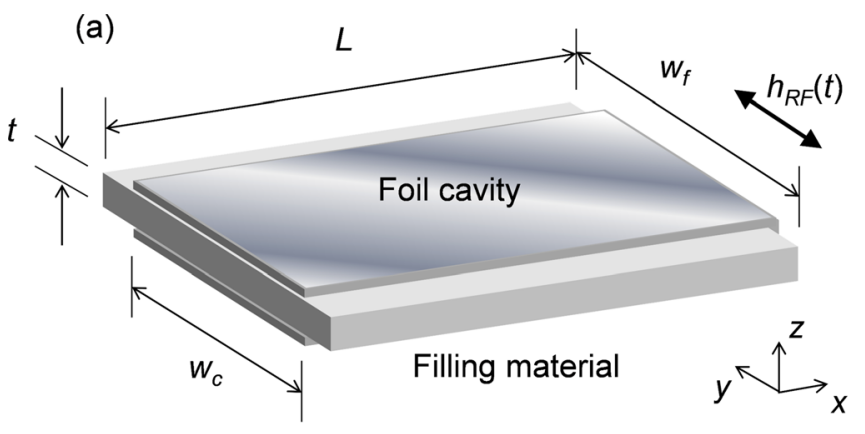

(b)

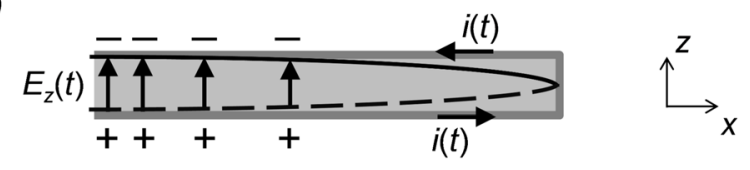

(c)

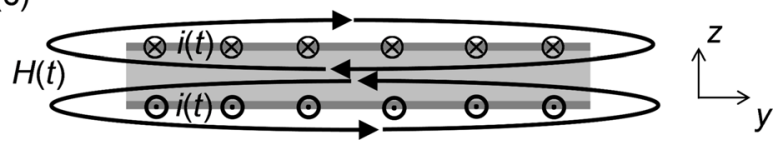

FIG. 1. (a) Schematic of a closed-end planar cavity formed from a metallic foil wrapped around the top, right end, and bottom surfaces of a filling material. In (b) the assumed the electric field $\left(E_{z}\right)$ configuration and charge distribution for the fundamental TEM mode in the $x-z$ plane is shown. The solid (dashed) black curve represents $E_{z}$ at a positive (negative) antinode for which $i(t)=0$. The resulting charge configuration is shown $(+$ and -$)$ for the solid black curve. $E_{z}$ is assumed to be uniform along the $y$ - and $z$-directions for a given position, $x$. In (c) the magnetic field $H(t)$ configuration in the $y-z$ plane resulting from the alternating current $i(t)$ is shown.

magnetic flux density is low, and the filling material within the cavity, where the flux density is high, Figure 1(c).

The detailed electromagnetic field distribution within the cavity is calculated by means of a finite element $\operatorname{method~}^{7}$ (FEM) model. The results of the experiments and of the FEM model are compared with Eq. (1) for a cavity of infinite width. Good agreement is only obtained for a non-permeable filling material. Finally we demonstrate that the cavity can be miniaturised by using a NiZn ferrite filler with a large $\operatorname{Re}\left(\mu_{r}\right)$ value.

The closed-end cavities consist of a metal foil wrapped around three surfaces of a filling material, Figure 1(a). The cavity length $L$ and thickness $t$ were set equal to the dimensions of the filling material while the cavity width $w_{c}$ was varied with respect to the fixed filling material width $w_{f}$. The cavity was excited using a uniform in-plane RF magnetic field $h_{R F}(t)$ generated by a $35 \mathrm{~mm}$ wide microstrip antenna placed beneath the cavity (not shown). The length of the microstrip was along the $x$-direction while the dominant RF magnetic field vector was along the $y$-direction, Figure 1(a). The same microstrip-cavity arrangement was also used in the FEM model.

A vector network analyser ${ }^{8}$ (VNA) was used to drive a RF current through the microstrip with frequency swept from $10 \mathrm{MHz}$ to $2 \mathrm{GHz}$. The frequency, linewidth, and absorption of the cavity were determined from a measurement of the transmitted power as a function of excitation frequency, i.e., a two-port measurement of the scattering matrix element S21. The interaction of the cavity with the microstrip antenna was investigated experimentally. ${ }^{9}$ For microstrip-cavity separations of $7 \mathrm{~mm}$ or greater, no significant variation of frequency was observed while the coupling efficiency to the cavity continued to decrease, indicating weak coupling. In order to minimise the interaction between cavity and microstrip, while maintaining sufficient signal level, all cavity testing was performed at a cavity-microstrip separation of $7 \mathrm{~mm}$.

The frequency dependent real and imaginary parts of $\varepsilon_{r}$ and $\mu_{r}$ for the teflon ${ }^{11}$ and ferrite filling materials were obtained from permeameter measurements similar to those described in Ref. 10 and are shown in Figures 2(a) and 2(b), respectively. The ferrite was a NiZn high frequency ferrite (Fair-Rite FR67) designed for applications at frequencies up to $50 \mathrm{MHz}$. The measured frequency dependence was subsequently used in the FEM model and analytical calculations. For teflon the frequency dependence of $\varepsilon_{r}$ and $\mu_{r}$ was weak with $\operatorname{Re}\left(\varepsilon_{r}\right) \sim 3$ and $\operatorname{Re}\left(\mu_{r}\right) \sim 1$, while $\operatorname{Im}\left(\varepsilon_{r}\right)$ and $\operatorname{Im}\left(\mu_{r}\right)$ were less than 0.2. In contrast, for the ferrite material $\mu_{r}$ showed a strong frequency dependence with $\operatorname{Re}\left(\mu_{r}\right)$ and $\operatorname{Im}\left(\mu_{r}\right)$ of the form first explained by Snoek. ${ }^{12,13}$ The initial (zero frequency) value of $\operatorname{Re}\left(\mu_{r}\right)$ was $\sim 30$ with a maximum value of $\sim 33$ at $105 \mathrm{MHz}$, and a monotonic decrease to $\sim 7$ at $600 \mathrm{MHz} \cdot \operatorname{Im}\left(\mu_{r}\right)$ also had a strong dependence on frequency ranging non-monotonically from $\sim 8$ to 16 between 100 and $600 \mathrm{MHz}$, increasing strongly at $105 \mathrm{MHz}$ where the maximum value of $\operatorname{Re}\left(\mu_{r}\right)$ was observed. For the ferrite $\operatorname{Re}\left(\varepsilon_{r}\right)$ was $\sim 8$ and had a weak dependence on frequency while $\operatorname{Im}\left(\varepsilon_{r}\right)$ was small, as for the teflon filling material. In Figure 2 anomalous behaviour is observed below $50 \mathrm{MHz}$ and $30 \mathrm{MHz}$ for the teflon and ferrite filling material, respectively. However, this artefact of the permeameter measurement is observed at frequencies outside of the range of measured cavity frequencies.

Figure 3(a) shows the measured $L$-dependence of the resonance frequency for teflon and ferrite filled cavities with dimensions $\left(L \times w_{f} \times t\right)$ of $L \times 12 \times 4 \mathrm{~mm}^{3}$ and $L \times 13 \times 3.2 \mathrm{~mm}^{3}$, respectively. The cavities were constructed using a $50 \mu \mathrm{m}$ thick aluminium foil, where $w_{c}=w_{f}$. A $25 \mu \mathrm{m}$ thick adhesive layer was used to fix the foil to the filling material to prevent any variation of the measured frequency due to movement of the foil. The adhesive formed less than $2 \%$ of the total volume within the cavity and had negligible influence on the resonant cavity mode. The experimental data for cavities filled with teflon and ferrite are

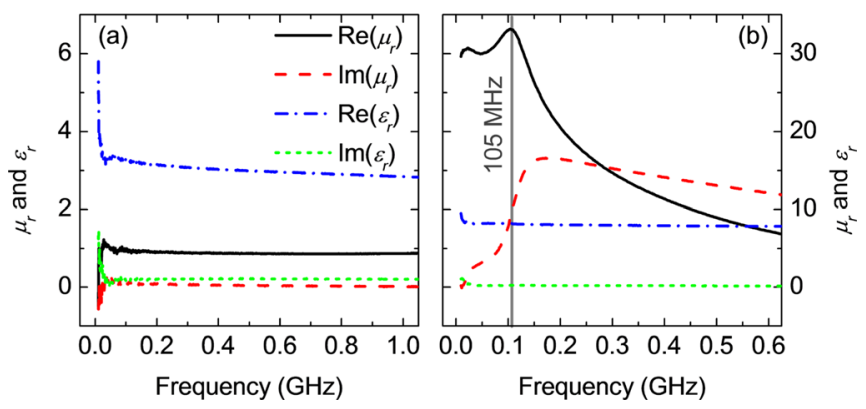

FIG. 2. Frequency dependence of the real and imaginary parts of the complex permittivity $\varepsilon_{r}$ and permeability $\mu_{r}$ of (a) the teflon, and (b) the ferrite filling material. The curves were calculated from two-port VNA measurements of the scattering matrix elements s11 and s 21 for a loaded stripline permeameter as described in Ref. 10. 

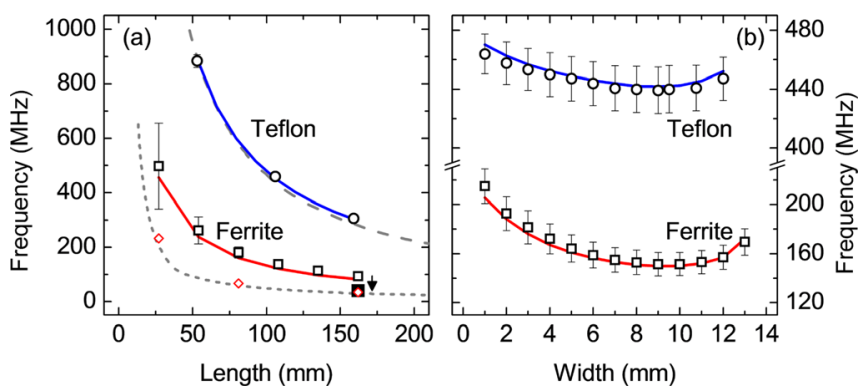

FIG. 3. Dependence of resonance frequency upon (a) length, and (b) width of the cavity. Measured frequencies for cavities filled with teflon (open circles) and ferrite (open squares) are overlaid with results from the FEM model (solid blue and red curves) and values calculated from Eq. (1) (dashed and dotted grey curves) that assume the measured values of $\varepsilon_{r}$ and $\mu_{r}$. The error bars represent the measured linewidth and are shown only when the linewidth is larger than the symbol size. In (a) resonance frequencies are also plotted for ferrite-filled cavities clad with the same ferrite. A single measured frequency (large filled square) and values obtained from FEM simulations (open red diamonds) are shown.

overlaid with the results of the FEM model and the values calculated from Eq. (1). For the teflon-filled cavities (with $\operatorname{Re}\left(\mu_{r}\right) \sim 1$ and $\left.\operatorname{Im}\left(\mu_{r}\right) \sim 0\right)$, the excellent agreement of the calculated frequencies with those obtained from the experiment and the FEM model demonstrates that the finite cavity width has only a weak influence on the cavity frequency, with only a small deviation $(<20 \mathrm{MHz}$ for $L=162 \mathrm{~mm}$ ) as $L$ is increased.

For the ferrite-filled cavities the results of the FEM model are also in excellent agreement with the measured frequencies. However there is a substantial discrepancy with the frequencies calculated from Eq. (1). Since the analytical model assumes an infinite cavity width, the magnetic field outside, above and below, the cavity is zero, and the permeability of the material outside the cavity does not enter the expression. The permittivity of the surrounding medium does not enter Eq. (1) because the electric field is largely confined to the interior of the cavity. While there is a finite end effect associated with electric field lines bowing out into the surrounding medium at the open end of the cavity, it is relatively small and can be neglected to a first approximation. However if the cavity instead has finite width, there must be finite magnetic field in the surrounding medium as shown in Figure 1(c). By a scaling argument, Eq. (1) is still approximately correct so long as the permeability of the filling material and the medium outside the cavity are the same. Therefore, for a cavity filled with a non-permeable dielectric and surrounded by air, Eq. (1) is a good approximation. When the cavity is filled with ferrite, Eq. (1) can still be applied if the surrounding space, or at least the regions close to the cavity where the magnetic field is significant, is also filled with the same ferrite material. However, for a ferritefilled cavity surrounded by air, the scaling argument breaks down and the finite width may significantly modify the resonance frequency.

To test this understanding a ferrite cavity with $L=162 \mathrm{~mm}$ and $w_{c}=10 \mathrm{~mm}$ was clad with the same ferrite material by placing ferrite blocks, of the same size as those used within the cavity, immediately above and below the cavity, so that the complex permeability inside and outside of the cavity was similar. The separation of $7 \mathrm{~mm}$ was maintained between the lower plane of the foil cavity and the excitation stripline. The measured cavity frequency was found to decrease to that calculated using Eq. (1), as shown in Figure 3 (arrow). FEM modelling of cavities that were both filled and surrounded by ferrite confirmed that the calculated resonance frequency of shorter cavities can also be recovered when $\mu_{r}$ is equal inside and outside of the cavity. However, for the shorter cavities the measured signal was too weak to detect due to shielding and magnetic losses (for $f>100 \mathrm{MHz}$ ) associated with the ferrite cladding.

The effect of cavity width $w_{c}$ upon resonance frequency was explored further by constructing additional cavities of different width, but on a filling material of fixed width. The dimensions of the teflon and ferrite filling materials were $106 \times 12 \times 4 \mathrm{~mm}^{3}$ and $81 \times 13 \times 3.2 \mathrm{~mm}^{3}$ respectively. Figure 3(b) shows that the resonance frequency decreases with increasing $w_{c}$, for both teflon (open circles) and ferrite (open squares) filling materials, until reaching a minimum value when $w_{c}$ is $\sim 3-4 \mathrm{~mm}$ smaller than $w_{f}$. The initial decrease in frequency occurs as the electric field is increasingly confined to the region between the conductors, so that the effective value of the permittivity in Eq. (1) is increased due to the presence of the filling material. However as $w_{c}$ approaches $w_{f}$ fringing electric fields at the edge of cavity begin to extend into the surrounding air, causing the effective permittivity to decrease again. The effect on the cavity frequency is more pronounced for materials with larger $\operatorname{Re}\left(\varepsilon_{\mathrm{r}}\right)$, i.e., for the ferrite filling material. In addition FEM modelling suggests that the dependence of frequency upon cavity width becomes stronger as the cavity length is reduced.

Figure 4 demonstrates that for a particular quarter wave resonance frequency, the length of the cavity can be decreased through the use of a ferrite filling material. Figure 4 shows the electric field configuration of ferrite $\left(27 \times 13 \times 3.2 \mathrm{~mm}^{3}\right)$ and teflon $\left(92.75 \times 12 \times 4 \mathrm{~mm}^{3}\right)$ filled cavities, with the same resonance frequency of $520 \mathrm{MHz}$, obtained from the FEM model. It is clear that the wavelength of the standing wave mode is reduced in the ferrite-filled cavity yielding a smaller effective cavity length. The $E$-field maps show that the $E$-field antinode is positioned slightly within the cavity rather than exactly at the open end. It is

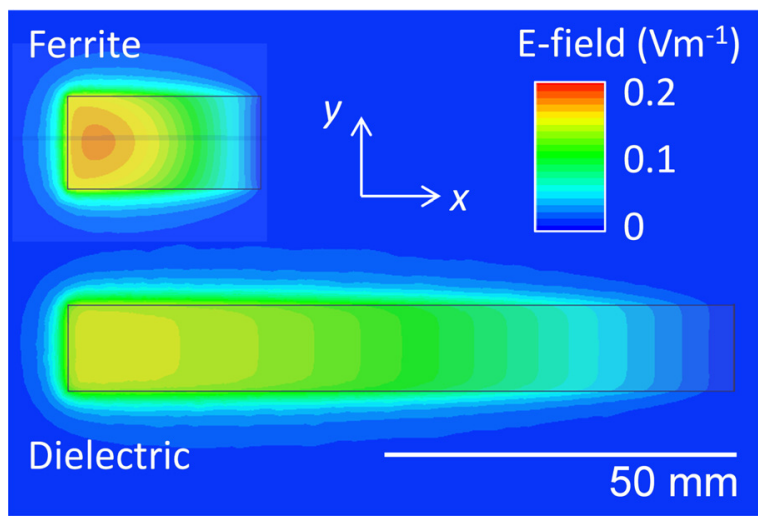

FIG. 4. Electric $(E)$ field maps generated by the FEM model for a ferrite and teflon filled cavity with the same frequency of $520 \mathrm{MHz}$ are shown. The maps represent the $z$-component of the $E$-field within the substrate across the $x-y$ plane equidistant from the upper and lower metallic planes of the cavity. 
expected that the electric field lines are concentrated in regions of high permittivity and so the effect is more pronounced in the ferrite material due to its larger permittivity value.

In summary we have constructed sub-wavelength closed-end planar cavities of finite width by wrapping aluminium foil on teflon and ferrite substrates. Comparison of the measured cavity frequencies with those obtained from a FEM model and analytical calculations allowed the effect of reduced cavity width upon the resonance frequency to be understood. Finally, it was demonstrated that a desired resonance frequency may be achieved with a ferrite-filled cavity of significantly reduced length. We anticipate that such ferrite-filled cavities may find immediate application in $\mathrm{HF}$ and ultra HF (UHF) tagging and RFID of conducting objects, within security, manufacture, and shipping environments, where the cavity may be excited by a magnetic field parallel rather than perpendicular to the plane of the cavity.

The authors gratefully acknowledge the financial support of the UK Engineering and Physical Sciences Research
Council (Grant No. EP/I500219/1), the University of Exeter Open Innovation Fund, and Crown Packaging UK PLC.

${ }^{1}$ D. Sievenpiper, L. Zhang, R. F. J. Broas, N. G. Alexopolous, and E. Yablonovitch, IEEE Trans. Microwave Theory Tech. 47, 2059 (1999).

${ }^{2}$ R. E. Collin, Field Theory of Guided Waves, 2nd ed. (Wiley-IEEE Press, 1990).

${ }^{3}$ J. A. Porto, F. J. García-Vidal, and J. B. Pendry, Phys. Rev. Lett. 83, 2845 (1999).

${ }^{4}$ Y. Takakura, Phys. Rev. Lett. 86, 5601 (2001).

${ }^{5}$ J. R. Suckling, A. P. Hibbins, M. J. Lockyear, T. W. Preist, J. R. Sambles, and C. R. Lawrence, Phys. Rev. Lett. 92, 147401 (2004).

${ }^{6}$ A. P. Hibbins, M. J. Lockyear, and J. R. Sambles, J. Appl. Phys. 99, 124903 (2006).

${ }^{7}$ HFSS, Ansoft Corporation, Pittsburgh, PA, USA.

${ }^{8}$ Agilent PNA-X N5242A $10 \mathrm{MHz}-26.5 \mathrm{GHz}$.

${ }^{9}$ See supplementary material at http://dx.doi.org/10.1063/1.4861844 for details of the effect of the cavity-microstrip separation on the measured frequency and absorption for teflon and ferrite filled cavities.

${ }^{10}$ W. Barry, IEEE Trans. Microwave Theory Tech. 34, 80 (1986).

${ }^{11}$ The measured complex parmittivity and permeability are within the expected range of teflon-like materials.

${ }^{12}$ J. L. Snoek, Nature 160, 90 (1947).

${ }^{13}$ J. L. Snoek, Physica 14, 207 (1948). 